\title{
Estimates of the ongoing need for social distancing and control measures post-“lockdown" from trajectories of COVID-19 cases and mortality
}

\author{
Mike Lonergan and James D. Chalmers
}

Affiliation: Division of Molecular and Clinical Medicine, School of Medicine, University of Dundee, Dundee, UK.

Correspondence: Mike Lonergan, Division of Molecular and Clinical Medicine, University of Dundee, Ninewells Hospital and Medical School, Dundee, DD1 9SY, UK. E-mail: m.lonerganđadundee.ac.uk

@ERSpublications

Data from multiple countries show that current restrictions on behaviour barely contain the spread of COVID-19. Resuming $20 \%$ of the currently excluded contact between individuals looks sufficient to restart the epidemic's progression in many countries. https://bit.ly/2B4dIOw

Cite this article as: Lonergan M, Chalmers JD. Estimates of the ongoing need for social distancing and control measures post-"lockdown" from trajectories of COVID-19 cases and mortality. Eur Respir J 2020; 56: 2001483 [https://doi.org/10.1183/13993003.01483-2020].

ABSTRACT By 21 May 2020, severe acute respiratory syndrome-coronavirus-2 (SARS-CoV-2) had caused more than 5 million cases of coronavirus 2019 (COVID-19) across more than 200 countries. Most countries with significant outbreaks have introduced social distancing or "lockdown" measures to reduce viral transmission. So the key question now is when, how and to what extent these measures can be lifted.

Publicly available data on daily numbers of newly confirmed cases and mortality were used to fit regression models estimating trajectories, doubling times and the reproduction number $\left(\mathrm{R}_{0}\right)$ of the disease, before and under the control measures. These data ran up to 21 May 2020, and were sufficient for analysis in 89 countries.

The estimates of $R_{0}$ before lockdown based on these data were broadly consistent with those previously published: between 2.0 and 3.7 in the countries with the largest number of cases available for analysis (USA, Italy, Spain, France and UK). There was little evidence to suggest that the restrictions had reduced $\mathrm{R}$ far below 1 in many places, with France having the most rapid reductions: $\mathrm{R}_{0} 0.76$ (95\% CI $0.72-0.82$ ) based on cases, and 0.77 (95\% CI $0.73-0.80$ ) based on mortality.

Intermittent lockdown has been proposed as a means of controlling the outbreak while allowing periods of increased freedom and economic activity. These data suggest that few countries could have even 1 week per month unrestricted without seeing resurgence of the epidemic. Similarly, restoring $20 \%$ of the activity that has been prevented by the lockdowns looks difficult to reconcile with preventing the resurgence of the disease in most countries.

This article has supplementary material available from erj.ersjournals.com

Received: 30 April 2020 | Accepted after revision: 26 May 2020

Copyright CERS 2020. This version is distributed under the terms of the Creative Commons Attribution NonCommercial Licence 4.0. 


\section{Introduction}

Coronavirus 2019 (COVID-19) is a respiratory disease caused by a novel coronavirus (severe acute respiratory syndrome-coronavirus-2 (SARS-CoV-2)) [1]. Its spread has already been the defining event of 2020 [1-3]. Two other coronaviruses, SARS-CoV and Middle East Respiratory Syndrome-CoV were identified in 2002 and 2012, respectively [4]. While both of these caused significant outbreaks of disease, they were more lethal and less transmissible, and did not cause pandemics.

SARS-CoV-2 has already spread through most of the world. And most countries have restricted travel, closed large parts of industry, restructured their economies, and focussed their efforts on its control. By 21 May 2020, 4927723 cases of COVID-19 had been confirmed by testing and 324737 of those individuals had died [5]. Cases have been reported in 207 countries.

Various measures have been adopted to control the spread of the virus. The most stringent of these has been termed "lockdown", and almost entirely restricted people to their own homes. The exact forms of the restrictions, and the exemptions allowed, have varied between countries and regions [6], but all have been expensive in terms of reducing economic activity and painful through restricting social interaction. The question of when and how far to ease the restrictions is, therefore, urgent [7,8]. At the end of April, some European countries began to try relaxing some of the restrictions but, in late May, there continued to be large numbers of mortalities in most parts of the world [5]. We therefore need to understand both the transmissibility of the virus and the effectiveness of the social distancing and lockdown measures that have been taken. The difference between the initial spread of the virus, before the restrictions were imposed, and its spread under lockdown gives an estimate of how effective lockdown measures have been. It can also suggest how far those measures can be relaxed without a resurgence of infection.

This paper uses publicly available data from multiple countries to model the spread of SARS-CoV-2, both before and under the lockdown, and estimate the scope for relaxing the current restrictions.

\section{Methods}

Data

Data on the numbers of new confirmed cases of COVID-19 and numbers of deaths reported for people known to have COVID-19 are available from the European Centre for Disease Prevention and Control website (www.ecdc.europa.eu/en/covid-19-pandemic). These were downloaded on the 22 May 2020. Mortality data was used for countries that had reported at least 100 deaths, and numbers of cases for those where at least 1000 confirmed cases had been reported. The pattern of arrival of infectious individuals determines the course of the initial stages of outbreaks, therefore days before a total of 10 deaths or 100 cases had been reported in each country were discarded. That left 89 countries with sufficient data for further consideration.

Both these types of data have important limitations: varying proportions of infections and deaths were not recorded in each country and over time. But they do provide a large, stable and standardised source of information that covers most countries. Confirmed cases are individuals who have tested positive for SARS-CoV-2. Unfortunately, no country has managed complete and continuous testing, and limitations and changes in testing strategies make this data particularly problematic. Data are skewed towards hospitalised cases and asymptomatic or paucisymptomatic cases, in particular, will be underreported. However, it is the best information on current prevalence, and recent changes in prevalence, that is available. The mortality data have an intrinsic lag that reduces their sensitivity to recent changes. Nevertheless, most countries have systematic testing of hospitalised cases and most mortality occurs in hospitals, so disease-specific mortality is potentially less biased than confirmed cases. The mortality data is therefore preferred in cases where the results conflict. Data for each included country were examined for inconsistencies and artefacts. The 10 occasions where countries reported negative numbers of cases were discarded. The data for China on 18 April 2020 showed a spike that appears to be an artefact of redefinition, and was also discarded. Iran reported nothing on the 4 April 2020 and a spike on the 5 April 2020 , so those reports were split evenly across the 2 days.

\section{Structure of the models}

The number of people a directly transmitted disease will infect each day is proportional to the number of infectious people multiplied by the number of susceptible people. While the absolute numbers of COVID-19 cases and deaths are shocking, the daily numbers are very small proportions of each country's population and, therefore, the numbers of susceptible individuals are almost constant. This means that, during periods of constant behaviour, exponential trajectories can be expected. And this should hold whether the daily numbers were increasing or decreasing. 
Each country introduced restrictions in a different pattern, so rather than attempting to interpret those rules and how people responded to them, the data were used to identify periods of steady increase and decline. The aim was to calculate exponential growth rates for each country before and under lockdown. This required identifying, and discarding, the transitional period where the data were affected by both the original, pre-lockdown and modified, lockdown behaviour.

The data were loaded into R 3.6.1 (R Project) [9] and mortality and cases from each country were analysed separately. Preliminary examination of the data showed that residuals around models fitted to almost all the datasets were over-dispersed relative to the Poisson distribution. All the models therefore used the negative binomial error family, with a log link function.

\section{Exponential models}

To find the best representation of the initial, exponential growth phase, a pair of generalised linear models were fitted for each combination of start and finish date at least 10 days apart such that the finish date was no later than 5 days before the day that the maximum number of events was observed. The 5-day interval was chosen after exploration of the Spanish and Italian data, where tight lockdowns were associated with obvious changes in the trajectory. These changes were particularly visible because of the large numbers of infections in those countries at that time. Adjusting this interval changes the estimates, and estimability, of individual countries' trajectories but produces similar overall patterns.

The first model of each pair simply contained a linear term for time. The second model contained both linear and quadratic terms for time. BIC, the Bayesian information criterion [10], was calculated and compared for each pair of models. The choice of BIC as the criterion for identifying exponential periods was entirely pragmatic. Exploratory analyses using Akaike's Information Criterion (AIC) [11], showed this selected very short intervals, and the small sample correction (AICc) worsens this problem. In BIC, the penalty for additional parameters is proportional to the natural logarithm of the number of data-points, so it is less eager to increase the complexity of models as sample size increases.

Linear models were considered potential representations of the initial exponential growth phase if they had: 1) positive point estimates of slope; and 2) BIC lower than their parallel quadratic model. If multiple models satisfied these criteria, the model where BIC was furthest below that of its quadratic equivalent was used.

For the exponential period under lockdown, the model was chosen in a similar way, but with its data not beginning before 5 days after the finish of the first exponential period. No requirement was placed on the slope of the second exponential period, so this could be negative or positive. It could even be larger than that for the first period, if that produced a better fit to the data. No explicit allowance for the lifting of restrictions was included because this would curve the trajectory, and such periods would therefore automatically be selected against. One exponential period was identified in the case data from 19 countries, and two exponential periods for 68 other countries. For the mortality data, these numbers were 1 and 53.

\section{Model checking}

To provide a visual check on these models, a generalised additive model (GAM) of the whole trajectory was also fitted [12]. In most cases, the ends of this curve are similar to the exponential models. It should be noted that GAMs favour steady change and curvature, while many of the changes in behaviour were quite sudden. For a few countries the mismatch between the GAM and exponential models gave, subjectively, cause for concern. These are indicated in the figures.

The slopes, and confidence intervals around them are of limited direct use. However, dividing the natural logarithm of 2 by them gives the doubling time of epidemic growth or halving time of its decay.

\section{Reproduction $(R)$ number}

$\mathrm{R}_{0}$, the basic reproduction number for the disease, is the expected number of people one infected person would pass the disease to in a naïve population. It is critical to disease spread: above 1 an epidemic will accelerate, below that the outbreak will fade away. Because these data include only a fraction of the cases in each country, they cannot be directly used to estimate $R_{0}$. Instead, the R0 library [13] was used to apply the method of WALLINGA and Lipsitch [14] to convert the estimates, and associated uncertainties, of the exponential trajectories into estimates of $\mathrm{R}_{0}$ both before and during lockdown. This approach requires an estimated distribution for the serial interval of infection. The log normal with mean 4 days and standard deviation of 2.9 days calculated by NisHIURA et al. [15] was used for this. While changing this distribution changes the individual estimated values for $R_{0}$, the relationship between the estimates before and during lockdown is relatively insensitive to plausible choices. 


\section{Estimates of the effect of "lockdown"}

Most discussion of $\mathrm{R}$ considers it as either indicating whether a disease outbreak will grow or fade away. At best the size of $\mathrm{R}$ is used as an indication of these changes. However, it is also a measure of how frequently individuals come into sufficiently close contact to pass the disease. The difference between the value of $\mathrm{R}$ and 1 therefore contains information on how much behaviour needs to change to stop the spread of a disease, or how much restrictions on contact between individuals can be relaxed without causing a resurgence in infections.

Four estimates of the scope for relaxing lockdown were then considered (table 1). The first, which will be called the time ratio, was a simple ratio of the exponential rates before and during lockdown. This indicates the number of days under lockdown that are required to balance a single day of previous behaviour. The second, the inverse of $\mathrm{R}_{0}$ on lockdown minus 1 , is the leeway. It is the proportion that contact under lockdown could be increased without causing a resurgence of the epidemic. As the continuing behaviour is quite different from that prevented, partly because much of what is permitted is within the domestic environment and most of that restricted is external to it, this measure is relatively uninformative and not discussed further. The third measure is the reclaimable fraction. It was calculated as:

$$
\left(1-\mathrm{R}_{\text {0lockdown }}\right) /\left(\mathrm{R}_{\text {0before }}-\mathrm{R}_{\text {0lockdown }}\right)
$$

Provided $\mathrm{R}_{\text {olockdown }}$ is less than both 1 and $\mathrm{R}_{\text {0before, }}$ this gives an estimate of the proportion of the behaviour, prevented by the lockdown, that can be resumed and result in an overall $R_{0}$ equal to 1 . The first and third approaches give different results because daily changes combine multiplicatively. An example that demonstrates this would be for a disease with a generation time of 1 week that had an $R_{0}$ of 2 initially and 0.5 under lockdown: alternate weeks of doubling and halving would oscillate around a constant value, with a mean contact rate of 1.25 , higher than the continuous $\mathrm{R}$ of 1 that would produce stability. Counterintuitively, that suggests more activity overall might be possible under a strategy of intermittent lockdown. Confidence intervals around each estimate were generated by drawing 1000 values from the relevant model parameter distributions.

While the current restrictions and lockdowns have very few precedents in public health, it is possible that similar approaches might be needed to control future outbreaks of other diseases. The current measures might be expected to reduce disease transmission by a similar proportion for other respiratory diseases. A fourth measure, the ratio of the two $\mathrm{R}$ values was, therefore, also calculated. This, the stop limit, is the maximum pre-lockdown $\mathrm{R}_{0}$ for a hypothetical disease or society where a lockdown of the current observed effectiveness, in proportion of contacts stopped, could halt an epidemic.

Results are presented for 89 countries. Because so many countries were considered, some results can be expected to appear significant by chance, and caution is needed in interpreting individual results. The discussion below will therefore largely focus on general patterns that show consistency across multiple countries.

\section{Results}

Figure 1 shows trajectories for the five countries with the highest number of deaths in this dataset, and table 2 shows parameter estimates for each of them. Equivalent graphs for the remaining countries are presented in the supplementary material. It can be seen that the intervals of exponential growth are in the early stages of the epidemic. That is consistent with the behavioural change to be expected, and that was

TABLE 1 Estimates of the scope for easing restrictions without causing a resurgence in the spread of SARS-CoV-2

\begin{tabular}{|c|c|c|c|}
\hline Name & Measure & Conditions & Interpretation \\
\hline $\begin{array}{l}\text { Reclaimable } \\
\text { fraction }\end{array}$ & $\left(1-R_{\text {olockdown }}\right) /\left(R_{\text {Obefore }}-R_{\text {olockdown }}\right)$ & $\mathrm{R}_{\text {Obefore }} \geqslant 1 \geqslant \mathrm{R}_{\text {olockdown }}$ & $\begin{array}{l}\text { The proportion of the contacts between individuals forgone under } \\
\text { lockdown that could be resumed without raising } \mathrm{R} \text { beyond } 1\end{array}$ \\
\hline
\end{tabular}

$\beta_{1} / \beta_{2}$ : slopes of the exponential periods before and under lockdown; $R_{0 \text { before }} / R_{0 l o c k d o w n}$ the reproduction number of coronavirus 2019 before and during lockdown. 

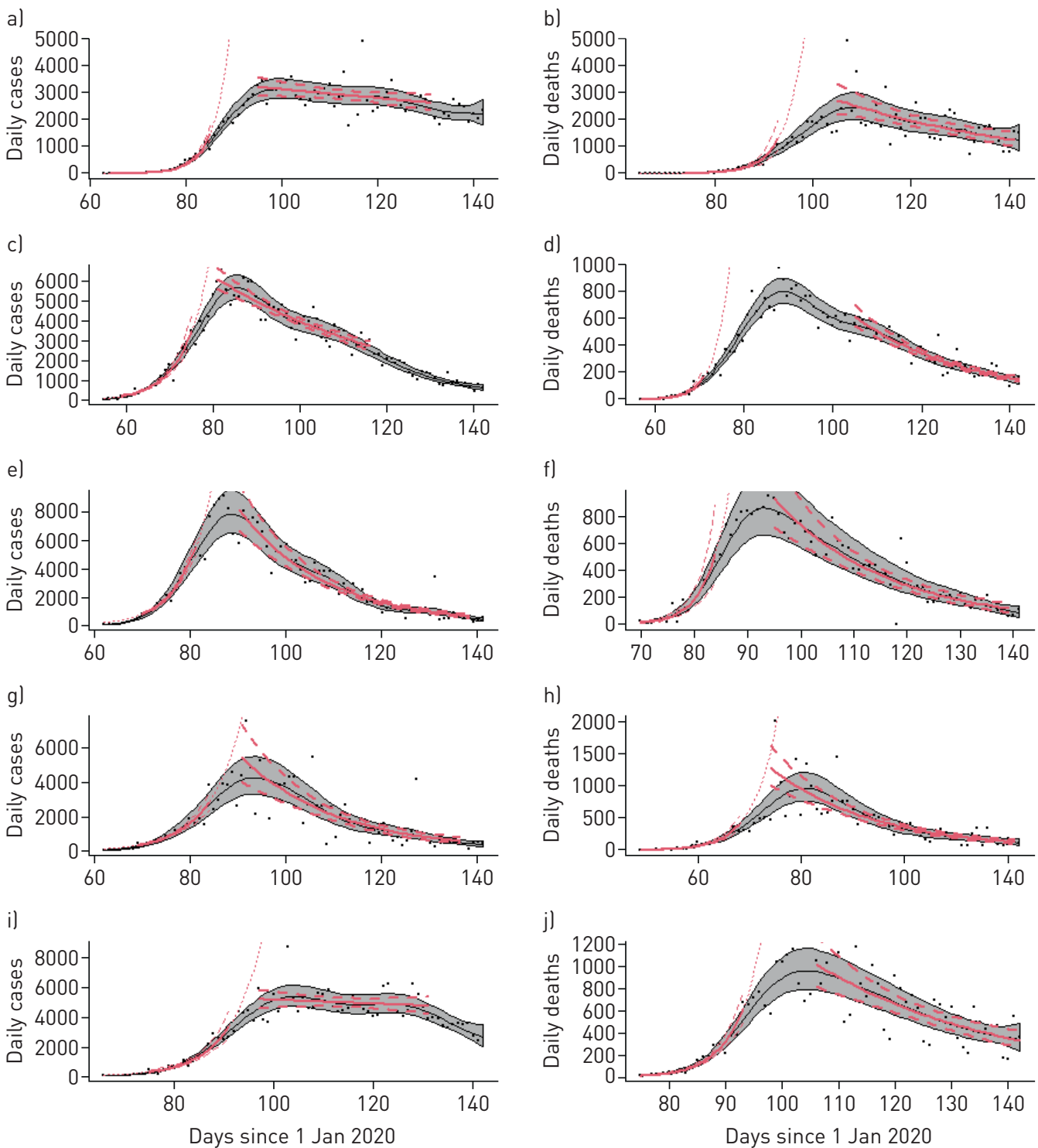

FIGURE 1 Trajectories of cases of and deaths from coronavirus 2019 (COVID-19). Data points represent numbers of $a, c, e, g, i)$ new cases or $b, d, f, h, j)$ deaths reported to the European Centre for Disease Prevention and Control for each day up to 21 May 2020 in a, b) USA, c, d) Italy, e, f) Spain, g, h) France and $i, j)$ UK. Grey shading represents the $95 \%$ confidence interval around a smooth trajectory (black line) estimated by a generalised additive model. Exponential patterns (mean and $95 \% \mathrm{CI}$ ) fitted to subsets of the data are in red. Details of the models are in the main text.

intended, as regulations were imposed and public awareness of the urgency of the COVID-19 problem spread. While it could be argued that the decline in Spanish cases appears to slow in early May, when they tentatively began easing their lockdown, this effect is small and removing this period produces very similar results.

For cases in the UK, the identified second exponential phase is almost flat, and followed by a decline that more nearly resembles the concurrent decline in mortality. This pattern probably results from dramatic increases in testing within the UK during April 2020. The confidence intervals around the exponential models are noticeably narrower than those around the GAMs, this is largely due to the information the GAMs require for the extra parameters that describe their curvature.

Visual inspection of the plots suggests that the method has selected subperiods that do not seem representative for cases in nine countries (Algeria, Bosnia, Canada, China, Greece, Iran, Moldova, Panama and Poland) and for mortality for two countries (Canada and Romania). In most cases these involve relatively small numbers of individuals or have their highest daily totals close to the end of the time-period. Trimming the data can resolve this problem for most of these countries, but would need to 
TABLE $2 \mathrm{R}_{0}$ estimated in the models of increasing and decreasing phases based on mortality data and confirmed cases of coronavirus 2019 up to 29 April 2020

\begin{tabular}{|c|c|c|c|c|c|c|}
\hline \multirow[t]{2}{*}{ Country } & \multicolumn{3}{|c|}{ Confirmed cases } & \multicolumn{3}{|c|}{ Deaths } \\
\hline & n & $\mathbf{R}_{0}$ before & $\mathbf{R}_{\mathbf{0}}$ after & n & $R_{0}$ before & $\mathbf{R}_{\mathbf{0}}$ after \\
\hline USA & 1551853 & $3.6(3.4-3.8)$ & $0.97(0.94-1.00)$ & 93439 & $3.2(2.7-3.7)$ & $0.89(0.84-0.94)$ \\
\hline UK & 248818 & $2.1(1.8-2.3)$ & $0.99(0.96-1.02)$ & 35704 & $2.6(2.4-2.9)$ & $0.85(0.80-0.90)$ \\
\hline Italy & 227364 & $2.2(2.0-2.4)$ & $0.89(0.87-0.91)$ & 32330 & $3.7(3.1-4.4)$ & $0.81(0.79-0.84)$ \\
\hline France & 143845 & $2.0(1.8-2.1)$ & $0.76(0.72-0.82)$ & 28132 & $2.7(2.4-3.0)$ & $0.77(0.73-0.80)$ \\
\hline Spain & 233286 & $2.2(2.1-2.4)$ & $0.74(0.71-0.78)$ & 27888 & $3.2(2.4-4.1)$ & $0.78(0.73-0.82)$ \\
\hline
\end{tabular}

Data are presented as $\mathrm{R}_{0}(95 \% \mathrm{CI})$.

be done differently for each one and introduce a subjective element into the analysis. For Canada, there are three apparently exponential periods, broadly similar to the pattern for cases in the UK. These results are included but indicated in the figures. The models ignored the first small outbreak in Singapore and picked up only the larger growth since then.

Table S1 contains the estimated slopes and standard errors for the two best exponential models of cases and mortality trajectories in each model. As doubling times are shown as these are more immediately interpretable (figure 2a). Many of these are too imprecise to be useful. However, for those countries with sufficient data, estimates of doubling times from mortality data are generally around 2-5 days. The estimates of halving time under lockdown (figure $2 \mathrm{~b}$ ) are generally $>7$ days, and much higher than the equivalent pre-lockdown doubling times. This impression is confirmed by inspection of individual trajectories, most of which decline more slowly than they increase.

The estimates of $\mathrm{R}_{0}$ contain essentially the same information as those of doubling times. The definition of the initial exponential period requires all the point estimates of $R_{0}$ to be $>1$, though the lower bounds of some of the confidence intervals fall below that threshold (figure $2 \mathrm{c}$ and table S1). Almost all the values under lockdown (figure $2 \mathrm{~d}$ ) are between 0.6 and 1.5, and the reductions in many countries are substantial (table 2). While previous versions of this analysis, using data up to 29 April 2020 showed little evidence for countries having reduced $\mathrm{R}_{0}<0.9$, the additional data available now suggests that values close to 0.75 have been achieved [16]. This suggests these countries have up to $33 \%$ leeway for the expansion of the permitted activities under lockdown, and that the stop limit for these lockdowns to prevent more contagious diseases is up to $25 \%$ higher than the initial $\mathrm{R}_{0}$ of COVID- 19 .

Because it is such a short time since most countries have introduced restrictions and changed the behaviour of their populations, there is a lot of uncertainty in the estimated trajectories under lockdown. But most of the more precise estimates (figure 3) suggest that periodical release of lockdown beyond 1 week in each month is very likely to result in the acceleration of the epidemic in many countries. And even this is based on the assumption that behaviour during intermittent releases from lockdown resembles typical behaviour in the period before the behavioural changes associated with the COVID-19 epidemics. It would be useful to understand what the countries where more time outside lockdown looks achievable have done differently.

Similarly, the reclaimable fraction of forgone contacts is $<0.2$ for most countries where reasonably precise data are available (figure 4). For 10 countries (Austria, Belgium, China, France, Hungary, Italy, the Netherlands, Spain, Turkey and the USA) models of both the case and mortality data exclude 0 from the 95\% confidence intervals around their estimates of the proportional easing of restrictions that would not raise $\mathrm{R}_{0}$ above 1 . For seven other countries, the mortality models capture their reported success in containing COVID-19, but the models of numbers of cases do not. In the figure, it can be seen that most of the confidence intervals around the estimates of the proportional easing in restrictions that would be consistent with avoiding a second peak of transmission extend to the left of the vertical line at 0.2 , and most of the more precise point estimates are also below that value. Resuming even $20 \%$ of currently prevented behaviour therefore looks extremely ambitious in most countries, with even a $10 \%$ easing appearing potentially risky in many of them.

\section{Discussion}

These analyses are, by their nature, provisional. They attempt to provide estimates and predictions from limited datasets. The models differ from most of the infectious disease models that have been applied to the COVID epidemics: they are based entirely on behaviour observed during the present pandemic of 


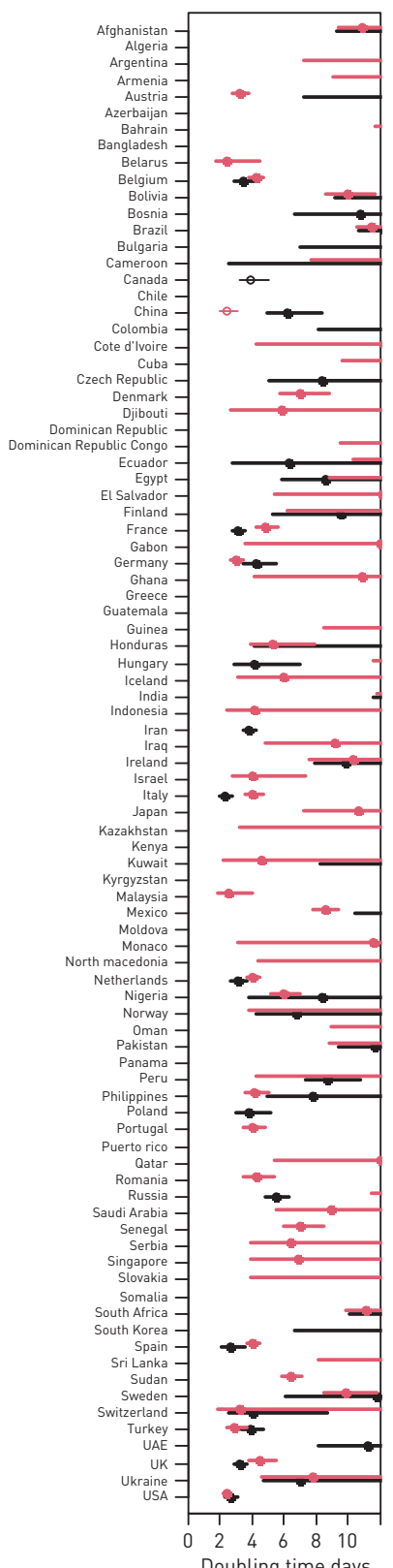

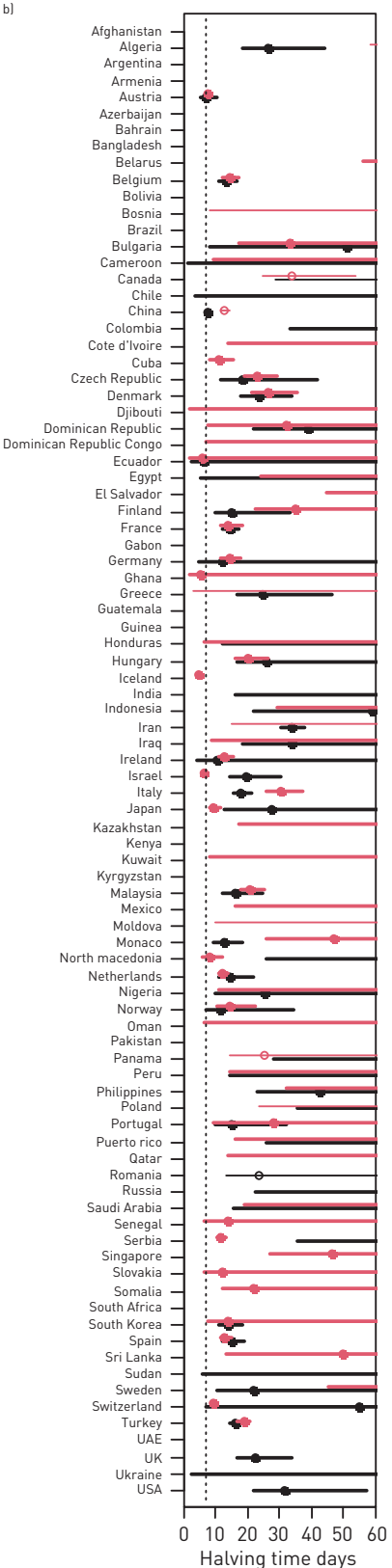

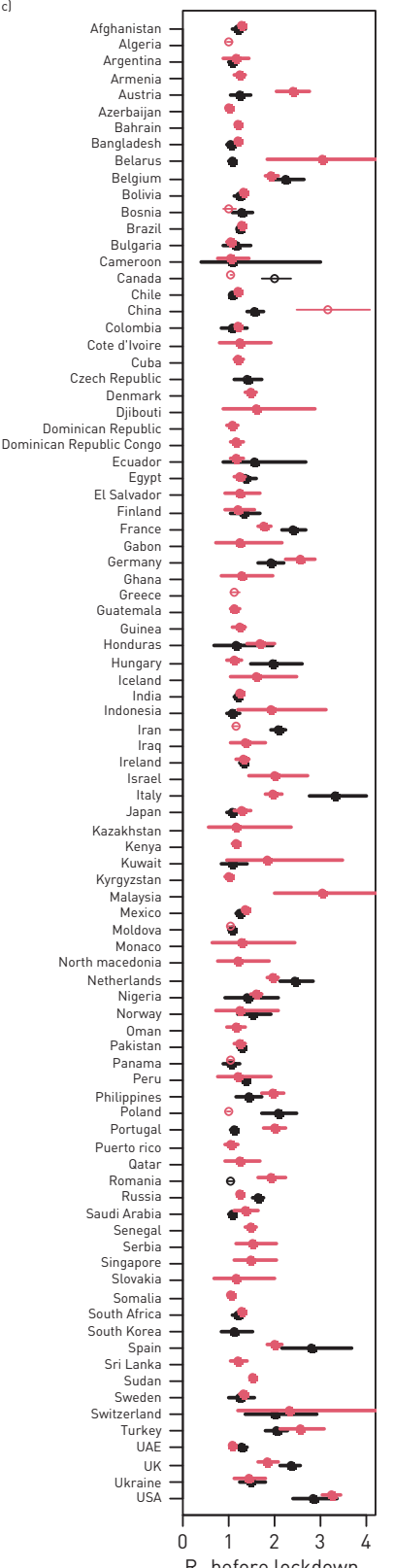

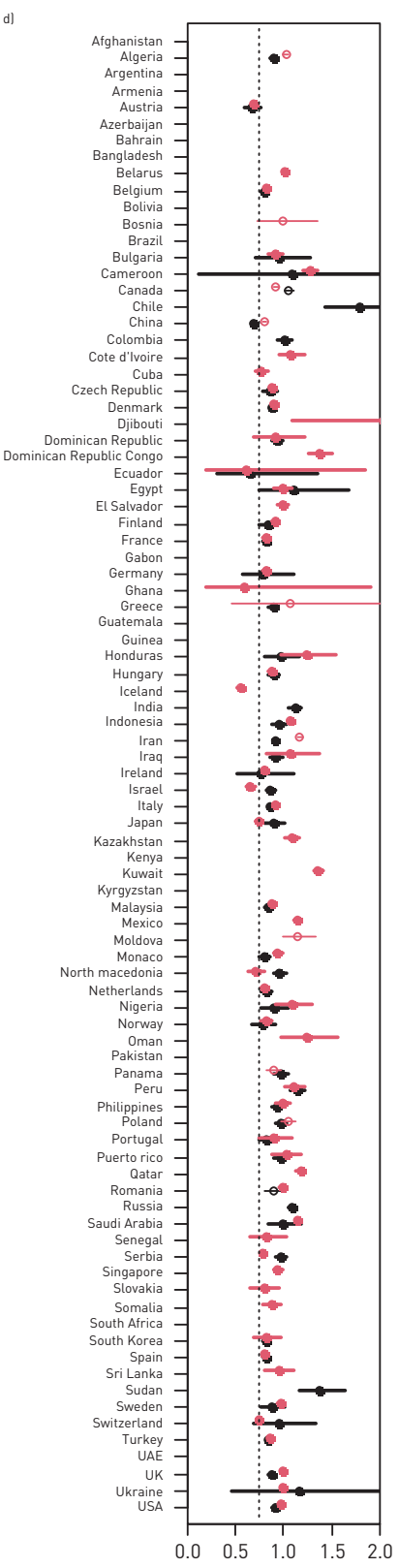

FIGURE 2 Doubling times and $R_{0}$ for exponential phases of outbreaks of coronavirus 2019 (COVID-19). Black data points are estimates based on mortality data, red data points are based on confirmed cases. Lines represent $95 \% \mathrm{Cl}$. Thin lines and hollow points indicate countries where plots of the modelled trajectories led to subjective doubts of the model fits. Gaps occur where an exponential phase was identified for only one of the data series. Table S1 contains all these data. a) Doubling time in the first (pre-lockdown) exponential phase of the epidemic. Lines that meet 0 indicate $a>2.5 \%$ chance that the epidemic was slowing over this period. b) Halving time in the second (locked down) exponential phase. The vertical dotted line is at 7 days and shows that almost all declines were slower than the preceding increases. c) The basic reproduction number $\mathrm{R}_{0}$, for COVID-19 in each country during the first exponential phase. d) $R_{0}$ under lockdown. The vertical dotted line at 0.7 is a guide to highlight how little evidence there is for lockdowns having reduced $R_{0}$ below this number.

SARS-CoV-2 and do not incorporate assumptions based on the behaviour and spread of other respiratory viruses. Only the conversion of the slope parameters to estimates of $\mathrm{R}_{0}$ uses a previously estimated distribution of generation times. This simplicity avoids reliance on the uncertain assumptions necessary for more traditional epidemiological models, instead increasing the confidence intervals around the estimates. And both the results and the confidence intervals it produces appear similar to many of those from far more computationally intensive methods. However, the simplicity does limit the details of human and viral behaviour, and the differences between countries, that can be resolved.

Despite this, a surprisingly clear picture is visible: if COVID-19 had been 25\% more transmissible the current lockdown measures would have been unable to halt the epidemic in Europe. However, in most 


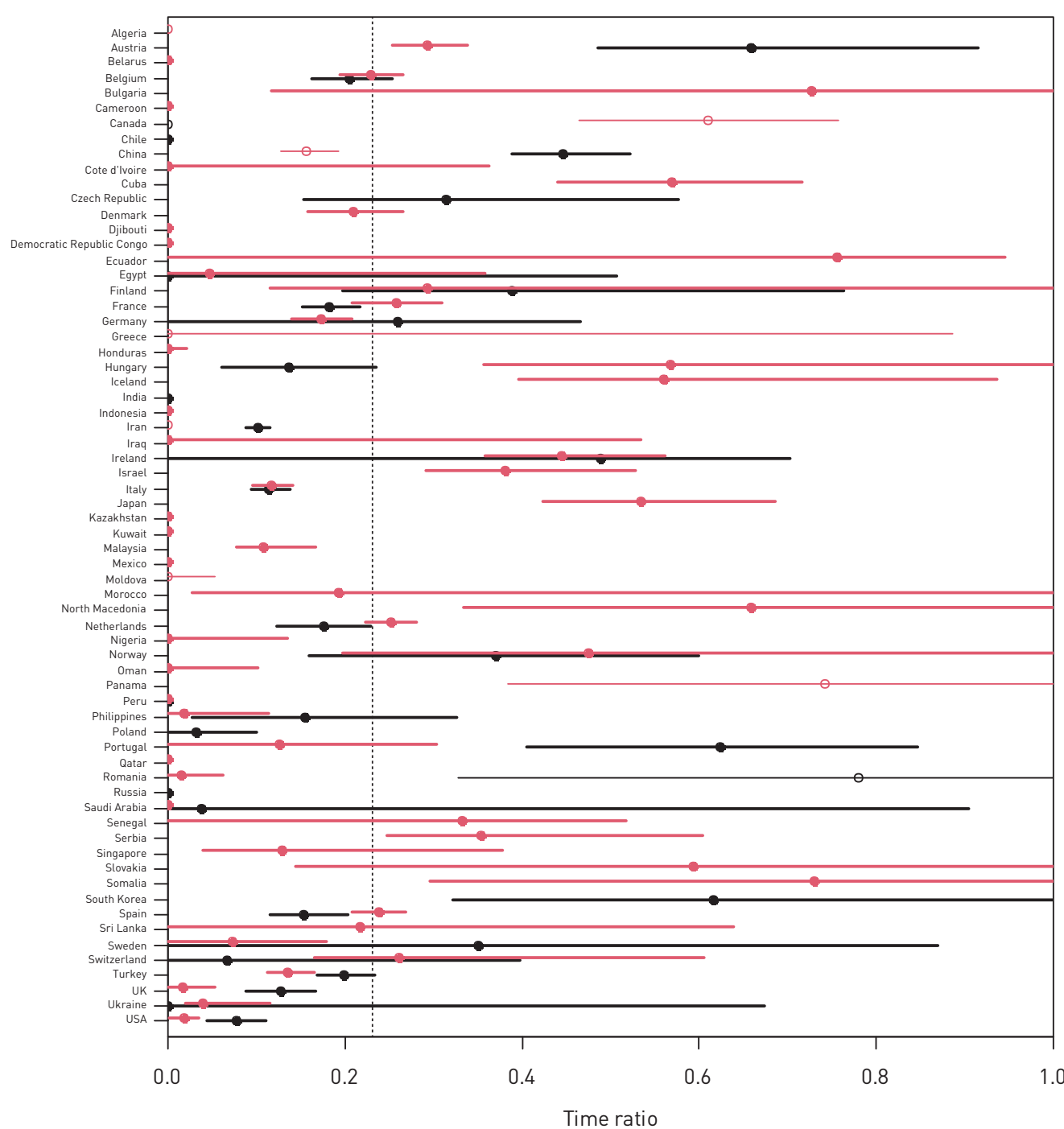

FIGURE 3 Estimated time ratios; the proportion of time countries could have in pre-lockdown state without the spread of severe acute respiratory syndrome-coronavirus- 2 resuming. For each country the exponential rate calculated before lockdown was divided by that under lockdown to give a mean and $95 \% \mathrm{Cl}$. Black data points represent mortality and red data points represent confirmed cases. Thin lines and hollow points indicate countries where plots of the modelled trajectories led to subjective doubts of the model fits. Lines that meet 0 indicate a $>2.5 \%$ chance that the epidemic was actually spreading faster over the second period than the first. The vertical dotted line indicates the effect of relaxing lockdown for 1 week (during which previous behaviour would be resumed) each month. Most of the more precise estimates lie to the left of this line, showing that there is little evidence that such a change would be consistent with stopping the epidemic.

data-rich countries its spread has been contained. Where it has been stopped, the margins for loosening the controls are limited: nowhere looks able to confidently resume half of what has been stopped. And the slow rates of decline in mortality suggest uncomfortable lower bounds on future mortality: sustaining a $10 \%$ per day decline, a rate faster than the best estimate for almost all the lasting declines in these data, implies that there will be a total of another nine times as many deaths to come as were reported yesterday; $5 \%$ per day increases that to 19 times.

There has been talk of lockdown being "a cure worse than the disease", but it is not a cure. At $5 \%$ per day it will take 35 days to claw back from 600 to 100 deaths per day; and another 45 to then get down to 10 . If $10 \%$ per day could be sustained it would only require 18 plus 20 days. Outside Europe, many countries are not yet clearly past, or even close to, the peak. That suggests easing in the near future will imply continuing mortality, and substantial easing is very likely to require the rapid re-imposition of lockdown.

$\mathrm{R}_{0}$, or more precisely $\mathrm{R}_{\mathrm{t}}$, seems to be the key to this problem: until and unless a vaccine or effective treatment becomes available, we need a liveable way to keep $\mathrm{R}$ below 1 . Lockdown, to a point beyond what most societies would have previously imagined accepting, can contain the disease's spread but it is hard to 


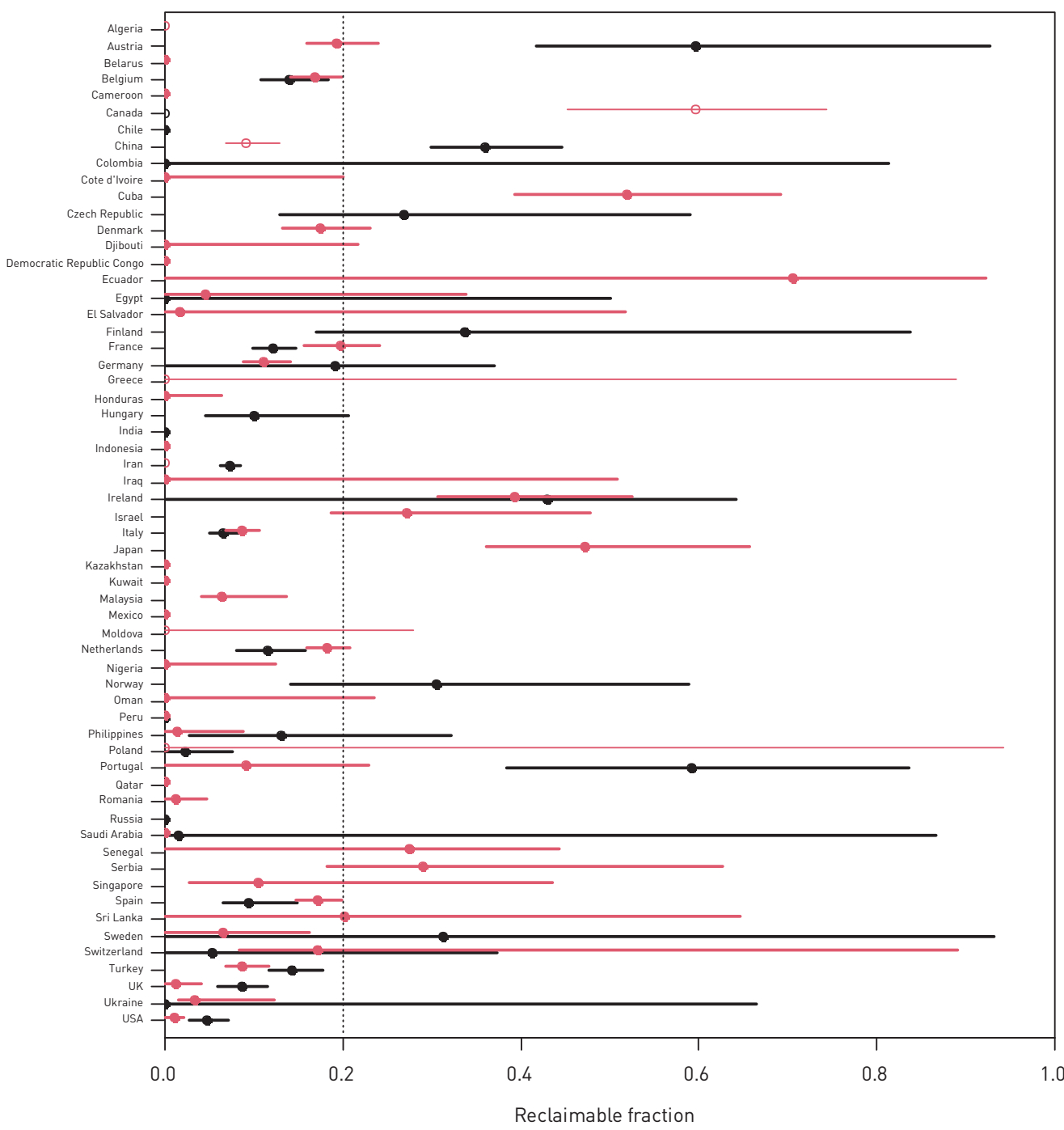

FIGURE 4 The scope for partial easing of lockdown while containing the spread of proportion of time countries could have in pre-lockdown state without the spread of severe acute respiratory syndrome-coronavirus-2. The reclaimable fraction, one minus $R_{0}$ under lockdown divided by the difference between the $R_{0}$ 's before and under lockdown, is an estimate of the proportion of the behaviour, that lockdown has prevented, that can be resumed without increasing $R_{0}$ past 1 , and restarting epidemic spread (table 1). Each row is the mean and $95 \% \mathrm{Cl}$ for a country. Black data points represent mortality data and red data points represent confirmed cases. Thin lines and hollow points indicate countries where plots of the modelled trajectories led to subjective doubts of the model fits. Lines that cut the left hand edge (0) indicate countries for whom lockdown may not yet have halted the epidemic. The vertical dotted line at 0.2 is a guide to highlight that there is little evidence for it being sustainable to resume $>20 \%$ of discontinued activity without the epidemic resuming its spread.

see it continuing indefinitely. These data suggest that unless a vaccine becomes rapidly available, discussions around exit strategy from current restrictions therefore need to move on from optimistic concepts of returning rapidly to normal activity. The data is more consistent with a need to adopting a "new normal" that can provide the optimal balance between allowing economic activity while ensuring very substantial reductions in prior social contacts (at least $80 \%$ reductions according to our best estimates). It is beyond the scope of this paper to describe what the components of a new normal may be but discussions will include continuing social distancing, public use of face-coverings, testing, tracking and isolating infected individuals and contacts and widespread screening of asymptomatic individuals among other considerations [17-19].

In summary, a simple analysis based on the behaviour of the SARS-CoV-2 pandemic to date across 89 countries suggests remarkably consistent effects of both exponential growth and slow decline in cases and mortality. Without a vaccine, these estimates are incompatible with a return to previous activities post "lockdown". 
Conflict of interest: M. Lonergan has nothing to disclose. J.D. Chalmers has received research grants from GlaxoSmithKline, Boehringer Ingelheim, AstraZeneca, Pfizer, Grifols, Bayer AG, Polyphor and Insmed; and received consultancy, congress travel or speaker fees from GlaxoSmithKline, Bayer Healthcare, Aradigm Corporation, Grifols, Pfizer, Boehringer Ingelheim, Napp and Insmed.

\section{References}

1 Zhu N, Zhang D, Wang W, et al. A novel coronavirus from patients with pneumonia in China, 2019. $N$ Engl J Med 2020; 382: 727-733.

2 Ghinai I, McPherson TD, Hunter JC, et al. First known person-to-person transmission of severe acute respiratory syndrome coronavirus 2 (SARS-CoV-2) in the USA. Lancet 2020; 395: 1137-1144.

3 Zhou F, Yu T, Du R, et al. Clinical course and risk factors for mortality of adult inpatients with COVID-19 in Wuhan, China: a retrospective cohort study. Lancet 2020; 395: 1054-1062.

4 Abo-Leyah H, Chalmers JD. Middle East Respiratory Syndrome: the need for better evidence in severe respiratory viral infections. Crit Care Med 2015; 43: 1344-1346.

5 European Centres for Disease Prevention and Control. COVID-19 situation update worldwide, as of 8 June 2020. www.ecdc.europa.eu/en/geographical-distribution-2019-ncov-cases Date last updated: 8 June 2020; date last accessed: 22 May 2020.

6 Flaxman S, Mishra S, Gandy A, et al. Report 13: Estimating the number of infections and the impact of non-pharmaceutical interventions on COVID-19 in 11 European countries. 2020. www.imperial.ac.uk/media/ imperial-college/medicine/mrc-gida/2020-03-30-COVID19-Report-13.pdf10.25561/77731 Date last accessed: 22 May 2020; date last accessed: 22 May 2020.

7 Prem K, Liu Y, Russell TW, et al. The effect of control strategies to reduce social mixing on outcomes of the COVID-19 epidemic in Wuhan, China: a modelling study. Lancet Public Health 2020; 5: e261-e270.

8 Cowling BJ, Ali ST, Ng TWY, et al. Impact assessment of non-pharmaceutical interventions against coronavirus disease 2019 and influenza in Hong Kong: an observational study. Lancet Public Health 2020; 5: e279-e288.

9 R Project. The R Project for Statistical Computing. www.r-project.org/ Vienna, Austria, R Foundation for Statistical Computing, 2019. Date last updated: 29 February 2020; date last accessed: 22 May 2020.

10 Schwarz G. Estimating the dimension of a model. Ann Stat 1978; 6: 461-464.

11 Burnham KP, Anderson DR, eds. Model Selection and Multimodel Inference: A Practical Information-Theoretic Approach. 2nd Edn. New York, Springer, 2002.

12 Wood SN. Generalized Additive Models: An Introduction with R. 2nd Edn. Boca Raton, CRC press, 2017.

13 Boelle PY, Obadia T. R0: Estimation of R0 and Real-Time Reproduction Number from Epidemics. R package version 1.2-6. https://cran.r-project.org/package=R0 Date last updated: 21 May 2015; date last accessed: 22 May 2020.

14 Wallinga J, Lipsitch M. How generation intervals shape the relationship between growth rates and reproductive numbers. Proc Biol Sci 2007; 274: 599-604.

15 Nishiura H, Linton NM, Akhmetzhanov AR. Serial interval of novel coronavirus (COVID-19) infections. Int J Infect Dis 2020; 93: 284-286.

16 Lonergan $\mathrm{M}$, Chalmers J. Estimates of the ongoing need for social distancing and control measures post-"lockdown" from trajectories of COVID-19 cases and mortality. medRxiv 2020; preprint [https://doi.org/10. 1101/2020.04.26.20080994].

17 Kissler SM, Tedijanto C, Goldstein E, et al. Projecting the transmission dynamics of SARS-CoV-2 through the postpandemic period. Science 2020; 368: 860-868.

18 Greenhalgh T, Schmid MB, Czypionka T, et al. Face masks for the public during the covid-19 crisis. BMJ 2020; 369: $\mathrm{m} 1435$.

19 Tobías A. Evaluation of the lockdowns for the SARS-CoV-2 epidemic in Italy and Spain after one month follow up. Sci Total Environ 2020; 725: 138539. 